\title{
Privatisierung, eine Antwort auf die Herausforderungen der Zeit
}

Andreas Zembaty

$» \mathrm{M}$ ehr Privat weniger Staat« ist als Kurzformel geeignet gesellschaftspolitische Tendenzen auch im Bereich der Kriminalpolitik zu beschreiben. Für die erfolgreiche Umsetzung im Rahmen der Sozialarbeit im Umfeld der Strafjustiz reicht die Formel allein jedoch nicht aus. Hier ist eine vorsichtige jedoch konsequente Organisationsentwicklung, die Chancen und Risiken abwägt, gefragt. Im Bereich der Straffälligen- und Opferhilfe kann in Österreich bereits über einen Zeitraum von mehr als 46 Jahren das Zusammenspiel zwischen privater Initiative, staatlicher bzw. gesetzlicher Aufgabendefinition und privater Durchführung in verschiedenen Entwicklungsstufen beobachtet werden. Das Zusammenwirken im Sinne einer gelungenen Zuständigkeits- und Aufgabenverteilung hat sich in diesem Bereich bewährt, weil im Laufe der Zeit eine Konzentration auf die jeweiligen Stärken erreicht wurde, wodurch Synergien genutzt und Doppelgleisigkeiten und Blockaden vermieden werden.

Die Entwicklungen der letzten Jahrzehnte im Bereich der österreichischen Straffälligen- und Opferhilfe bei »NEUSTART « und im Zeitraffer dargestellt:

\section{Dienstleistungen}

Die einstmals semiprivate Vereinigung zur Durchführung der Bewährungshilfe primär für Jugendliche, hat zunehmend weitere Aufgaben der Straffälligenhilfe übernommen und den Wirkungsbereich der Organisation auf das gesamte Bundesgebiet ausgedehnt. Ab 1978 wurden als Antwort auf Mängel der allgemeinen Sozialhilfe der Länder für Haftentlassene eigene niederschwellige Angebote aufgebaut, für die inzwischen die staatliche Förderung gesetzlich verankert ist. Die größte Aufgabenerweiterung in quantitativer und qualitativer Hinsicht brachte die "Erfindung «, modellhafte Erprobung und schließlich gesetzliche Einführung des außergerichtlichen Tatausgleichs für jugendliche und erwachsene Beschuldigte seit den Achtzigerjahren. Damit trat auch die Opferperspektive in den Fokus der institutionellen Methodenentwicklung. 1999/2001 folgte das Angebot für schwer beeinträchtigte Verbrechensopfer, die nunmehr proaktiv betreut werden. Im Zusammenhang mit dem sogenannten Diversionsgesetz 2000 wurde die Vermittlung gemeinnütziger Leistungen, Schulungen, Kurse und Betreuung als Sank- tionsalternative übernommen. Präventionsmaßnahmen von der Schulsozialarbeit, Jugendhilfe, Suchtprävention bis zur Online Beratung wurden in den letzten Jahren entwickelt.

\section{Organisation}

Die Übertragung der Bewährungshilfe an einen Verein war in Österreich zunächst provisorisch und befristet. Die Verstaatlichung war gesetzlich vorgesehen, wurde jedoch mehrmals aufgeschoben, ehe die zeitliche Begrenzung der "privaten Durchführung « in einer BewHG-Novelle 1980 fiel. Ein höheres Maß an Planungssicherheit und Autonomie für den Verein sicherte aber erst eine Statutenreform und der Abschluss eines »Generalvertrags « mit dem Bundesministerium für Justiz im Jahre 1994. Erst danach wandelte sich die Organisation zu einem Betrieb mit der notwendigen Personalhoheit und Kollektivvertragsfähigkeit, mit modernem Arbeitsrecht und Finanzwesen.

Den jüngsten Abschluss der Organisationsreform 2003 bildete eine Zusammenführung der Einrichtungen für verschiedene Dienstleistungen in 15 größeren regionalen Leistungszentren. Durch den neuen Vereinsnamen "NEUSTART « und ein neues Erscheinungsbild, soll auf die Positionierung »Hilfe schafft Sicherheit « und auf das umfassende Angebot der Straffälligen- und Opferhilfe aufmerksam gemacht werden. Diese so auch geschaffene »schlankere « und flexiblere Organisation ist nicht zuletzt als Antwort auf Restriktionen der öffentlichen Haushalte und auf den Zwang zu verstehen, eine neuartige "Marktsituation", (europaweite) Konkurrenz und weitestgehende Abhängigkeit von einem öffentlichen Auftraggeber zu bewältigen.

Der Paradigmenwechsel seit den 90iger Jahren ist durch

- Verstärkte Anspruchsgruppenorientierung:

- Kunden (Auftraggeber, Zuweiser)

- Klienten

- Öffentlichkeit: extern (Medien,...) intern (Mitarbeiter/innen)

- Weiterentwicklung des sozialarbeiterischen Angebotes,

- betriebswirtschaftliche Kompetenz im Management der Sozialarbeit und

- professionelle Dokumentation im Spannungsfeld zum Datenschutz

gekennzeichnet.
Am Beispiel des Vereins "NEUSTART« lassen sich die wesentlichen Kriterien zeigen, die aus meiner Sicht erfüllt sein müssen, um die Chancen der privaten Durchführung öffentlicher Aufgaben im Sozialbereich im Sinne einer optimalen Umsetzung des von der öffentlichen Hand definierten gesellschaftlichen Auftrags zu nutzen ohne Beeinträchtigungen einer adäquaten Versorgung der KlientInnen mit sozialen Leistungen und der berechtigten Interessen der in diesem Bereich beschäftigten Personen in Kauf zu nehmen:

\section{Öffentlichkeit - Definition des Auftrags, Qualität, Bestandsgarantie:}

Wesentliche Grundlage für erfolgreiche Privatisierung ist die Sicherstellung der notwendigen Rahmenbedingungen, eine klare gesetzliche Grundlage einschließlich der inhaltlichen Beschreibung der gewünschten Leistung und der Übernahme der Finanzierung durch die öffentliche Hand. Die Ausgestaltung des jeweils den Klienten anzubietenden Leistungsumfangs darf im Kern nicht ausschließlich der wirtschaftlich, ideologisch oder sonst wie motivierten Entscheidung des privaten Anbieters überlassen werden, sondern ist vom zuständigen Organ der öffentlichen Verwaltung mitzubestimmen. Es ist sicher zu stellen, dass allen grundsätzlich leistungsberechtigten Klienten, unter gleichen Voraussetzungen, die gleichen Leistungen zukommen und so ein Anspruch auf allgemeine Qualitätsmerkmale besteht.

Die Forderung nach gesetzlicher Definition und Absicherung von Zielrichtung und Ausmaß sozialer Leistungen und die Sicherstellung schließt eine grundsätzlich längerfristige Bestandsgarantie ein. Dies ist jedenfalls für alle Angebote notwendig, welche auf die Bewältigung gesellschaftlicher und individueller Problemlagen abzielen, die nicht durch kurze isolierte Interventionen erfolgreich bearbeitet werden können. Aufgabe der Institution ist es aufgrund der Auftraggeber-, Zuweiser- und Klienteninteressen das eigene Angebot adäquat auszurichten. Angesichts der meist unterschiedlichen Interessenlagen stellt das eine besondere Herausforderung für das Sozialmarketing von "NEUSTART « dar, sollen auch noch die Ansprüche der (ver)öffentlichten Meinung integriert werden. 


\section{Organisation}

Chancen sind im einheitlichen öffentlichen Auftritt für die Interessen der KlientInnen und der Organisation, der relativ hohen Autonomie in fachlichen und organisatorischen Fragen, der einheitlichen Durchführung der sozialarbeiterischen Leistungen im Bundesgebiet, der Möglichkeit Zusatzfinanzierungen zu generieren, der Schaffung fairer Arbeitsbedingungen für MitarbeiterInnen und der Transparenz bezüglich der sozialarbeiterischen Leistungen und der Kosten $\mathrm{zu}$ sehen. Private Organisationen können schneller und flexibler auf die Änderungen der Ansprüche von Umwelten reagieren.

Risiken sind, dass erfahrungsgemäß öffentliche Finanzierungsengpässe relativ rasch auf die Gesamtorganisation durchschlagen, Entwicklungen im Bereich der Ausgliederungen von öffentlichen Leistungen das Potenzial von Konkurrenz forcieren, die Arbeitsplatzsicherheit im öffentlichen Dienst unvergleichbar ist und die Abhängigkeit von parteien- und ideologiegesteuerten Ansprüchen relativ hoch ist.

Für das Management ergeben sich, durch Vorteile der Privatisierung durch eine größere Handlungsfähigkeit des Managements, eigenständige Prioritätensetzung und ein vermehrter Spielraum bei Budgeteinsparungen durch interne Steuerungsmaßnahmen.

\section{Kunden}

Der Auftraggeber Justiz und die Zuweiser können letztlich höhere Qualität und raschere Anpassung an Umweltveränderungen, als dies in staatlichen Bürokratien möglich ist, vom »Privaten « erwarten. Darüber hinaus ist durch die Vertragsgestaltung, und den dort vereinbarten Kontrollmöglichkeiten, das Risiko Fehlentwicklungen machtlos gegenüber zu stehen, sehr gering.

Privatisierungsvorteile für Zuweiser (Richter, Staatsanwälte, etc.) ergeben sich durch eine bessere Kontaktmöglichkeit - eine Adresse, eine Ansprechperson für alle Dienstleistungen - durch ein zuweiserorientiert agierendes Leitungsteam, durch die regelmäßige Evaluation der Zuweiserzufriedenheit und durch den verstärkten Kontakt mit den Sozialarbeitern. Mögliche Nachteile für Zuweiser sind in der Irritation durch neues Erscheinungsbild (neuer Name, neues Logo), neue Leitungspersonen, veränderte Standorte und Organisationsabläufe zu beachten.

\section{Klienten}

Chancen für Klienten/Klientinnen sind in der allgemeinen Haltungsänderung durch die Aufwertung der Klienten als Anspruchsgruppe zu sehen. Weitere Vorteile: Professionalisierung, Serviceorientierung bei Erstkontakt (Telefon,etc.), Betonung der Klientenrechte, Einsparungen auf Over- headbereich beschränkt und vor allem genaue Indikationsstellung um richtige Leistung effizient angeboten zu bekommen.

\section{Mitarbeiter}

Die MitarbeiterInnen sind bei Erbringung sozialer Dienstleistungen der größte Kostenfaktor und damit in Einklang auch die wichtigste Ressource. Das in ausgegliederten Bereichen anzuwendende private Arbeitsrecht bietet Instrumente, welche wesentlich flexiblere Regelungen für unterschiedliche Anforderungen wie auch hinsichtlich der Möglichkeit auf Veränderungen der Anforderungen und Umweltbedingungen im Zeitablauf zu reagieren, ermöglichen.

Neben diesen eher arbeitgeberseitig wirksamen Vorteilen sollen private Anbieter sozialer Dienstleistungen auch ihren Angestellten faire Arbeitsbedingungen und ausreichende Sicherheit der Arbeitsplätze bieten. Es ist nicht nur eine Frage politischer Korrektheit, die Ausgliederung der Durchführung öffentlicher sozialer Dienstleistungen an private Träger nicht zur Durchsetzung genereller Verschlechterungen der Arbeitsbedingungen zu nutzen. Dies zu vermeiden ist auch wesentliche Voraussetzung für die Sicherung und Weiterentwicklung der Qualität der zu erbringenden Leistungen.

Chancen für Mitarbeiter sind die bessere Bezahlung durch Angestelltenverhältnis, die Erhöhung der Arbeitsplatzsicherheit durch größere Organisationseinheiten, die Verbesserung der technischen Ausstattung, die Arbeitsplatzqualität (Fortbildungsangebote, Ressourcen für Klientenarbeit) auf hohem Niveau, die vermehrten Aufstiegschancen und der vermehrte fachliche Austausch.

Risiken für Mitarbeiter können anfänglich Irritationen, Unsicherheit durch weitreichende Veränderungen, neue Teamzusammensetzung und damit verlangte Neuorientierung, Verunsicherung durch Anforderung nach mehr Flexibilität, vereinheitlichte Standards welche das Ende von »Individuallösungen« bedeuten, Neuausschreibung der Leitungsfunktion, Bestellung auf Zeit und Bewertungsfragen (Beamtendienstrecht, Zulagen,etc.) sein.

Insgesamt erscheint nach den bisherigen Erfahrungen die Übertragung öffentlicher, sozialer Aufgaben im Bereich der Kriminaljustiz an private Träger ein erfolgversprechendes Modell zusein. Chancen für Auftraggeber, Mitarbeiter, Klienten und die Öffentlichkeit sind gegeben. Wichtig ist während des Prozesses Risiken und Interessenskonflikte zu benennen und dort, wo es möglich ist, auch für einen Ausgleich zu sorgen.
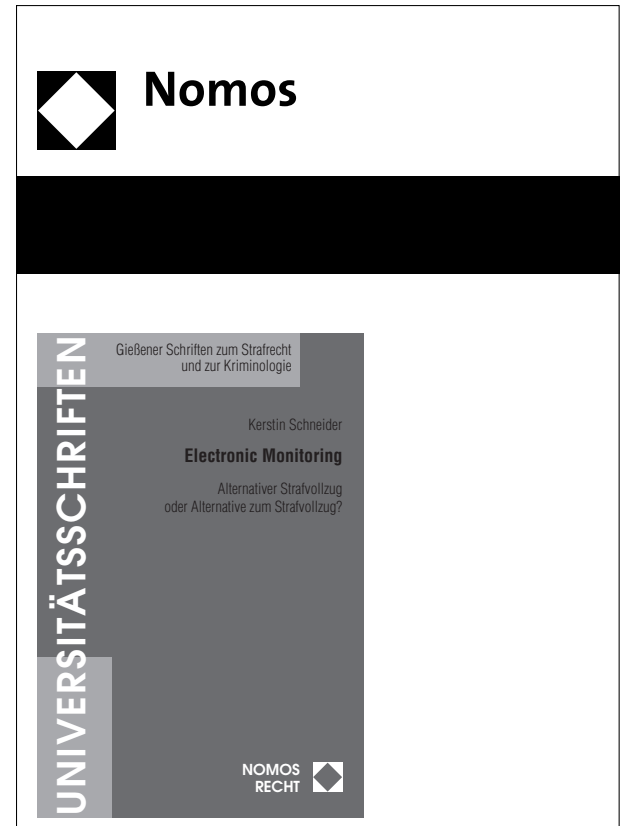

\section{Electronic Monitoring}

Alternativer Strafvollzug oder Alternative zum Strafvollzug?

Von Kerstin Schneider

2003, 236 S., brosch., 46,- €, ISBN 3-8329-0268-6

(Nomos Universitätsschriften - Recht / Unterreihe: Gießener Schriften zum Strafrecht und zur Kriminologie, Bd. 6)

In Zeiten chronisch überfüllter Justizvollzugsanstalten und leerer Haushaltskassen wird nach Alternativen gesucht, um eine Reduzierung der Belegungszahlen in den Gefängnissen zu erreichen und gleichzeitig Kosten einzusparen. Große Hoffnungen werden auf den Einsatz des elektronisch überwachten Hausarrestes (sog. Fußfessel) gesetzt. Während diese Sanktionsform in den USA bereits fest in das Sanktionssystem integriert ist, werden in Deutschland derzeit erste Erfahrungen mit Hilfe eines Modellversuches gewonnen.

Die Autorin stellt die rechtliche Ausgangslage in den USA und die praktische Ausgestaltung der elektronischen Überwachung im Gerichtsbezirk von San Francisco/Kalifornien dar, um sodann anhand einer rechtsvergleichenden und praxisorientierten Analyse die Möglichkeiten eines Einsatzes in Deutschland zu überprüfen.

Bitte bestellen Sie bei Ihrer Buchhandlung oder bei: Nomos Verlagsgesellschaft $\cdot 76520$ Baden-Baden Telefon o 72 21/21 04-37/-38 • Telefax 072 21/21 04-43 sabine.horn@nomos.de•www.nomos.de 